\title{
CARCINOMA SARCOMATOIDE DE RIÑÓN. APORTACIÓN DE UN NUEVO CASO, Y REVISIÓN DE LA BIBLIOGRAFÍA
}

\author{
I. GÓMEZ GARCÍA, F.J. BURGOS REVILLA, E. SANZ MAYAYO, \\ R. RODRÍGUEZ PATRÓN, S. CONDE SOMESO*, M. GARCÍA COSIO PIQUERAS**, \\ D. GARCÍA ORTELLS, E. FERNÁNDEZ FERNÁNDEZ, Á. ESCUDERO BARRILERO
}

\begin{abstract}
Servicio de Urología. *Servicio de Cirugía General y Aparato Digestivo. ${ }^{* * S e r v i c i o ~ d e ~ A n a t o m i ́ a ~ P a t o l o ́ g i c a . ~}$ Hospital Ramón y Cajal. Madrid.
\end{abstract}

Actas Urol Esp. 27 (8): 649-653, 2003

\section{RESUMEN}

CARCINOMA SARCOMATOIDE DE RIÑÓN. APORTACIÓN DE UN NUEVO CASO, Y REVISIÓN DE LA BIBLIOGRAFÍA

Se presenta un nuevo caso de carcinoma renal sarcomatoide, en un varón de 67 años con recidiva locorregional a los 4 meses de la cirugía, con dos episodios de hemorragia retroperironeal tras la segunda intervención. El objetivo de este trabajo es aportar un nuevo caso y hacer una revisión de la bibliografía.

PALABRAS CLAVE: Carcinoma fusocelular. Carcinoma sarcomatoide renal. Sindrome de Wünderlich.

\section{ABSTRACT}

RENAL SARCOMATOID CARCINOMA. A NEW CASE CONTRIBUTION AND BIBLIOGRAPHIC REVISSION

We report a new case of sarcomatoid renal cell carcinoma, in a male of 67 years old, with locoregional recidive four months after first surgery, with two episodes of retroperitoneal hemorrhage after second surgery. The objective of this work is to contribute a new case and of doing one revision.

KEY WORDS: Fusocelular carcinoma. Sarcomatoid renal cell carcinoma. Wunderlich sindrom.

$\mathrm{E}^{\prime}$ carcinoma sarcomatoide de riñón es un tumor infrecuente en torno al 1 y al 6\%, según las series de las neoplasias malignas renales ${ }^{1,4,5}$. Su clínica es muy similar a cualquier patología maligna retroperitoneal, presentando manifestaciones inespecíficas y sensación de masa. El diagnóstico suele ser con la enfermedad avanzada; siendo la cirugía el tratamiento de elección en las formas localizadas. Es un tumor con mal pronóstico, siendo la supervivencia media de seis meses.

$\mathrm{El}$ interés de este trabajo es aportar un nuevo caso de este raro tumor, y hacer una revisión exhaustiva tanto de la bibliografía internacional y de la nacional.

\section{CASO CLÍNICO}

Varón de 67 años de edad que ingresa en el servicio de medicina interna por síndrome constitucional, anemia y masa en flanco derecho.

Como antecedentes personales de interés, destacan: accidente cerebro vascular en territorio de la cerebral media, secundaria a fibrilación auricular a los 64 años; enfermedad pulmonar obstructiva crónica; tratamiento habitual con dicumarínicos orales y amiodarona.

El paciente presenta anorexia, astenia, y pérdida de 12 kilogramos de peso en 4 meses. Veinte días previos al ingreso, se inicia un dolor súbito en flanco y fosa renal derecha. La anamnesis urológica es normal. En la exploración física se palpa 
en hemiabdomen derecho, masa dolorosa. Resto de la exploración anodina. En el estudio analítico, destaca anemia normocítica y normocrómica (hemoglobina 10,6), y prolongación de la VSG; el estudio bioquímico, urinario y de marcadores tumorales normal. La ecografía abdominal objetiva, masa renal derecha con patrón ultrasonográfico mixto de 17 x 13 centímetros de diámetro, con un área extraparenquimatosa compatible con hematoma. La tomografía axial computerizada de cuerpo completo (T.A.C.) evidenció masa mixta de predominio sólido en polo superior de riñón derecho, con probable infiltración del músculo psoas; no se objetivó adenopatías, ni trombosis vascular (Fig. 1). Previo a la cirugía, y dado el gran tamaño de la masa tumoral se procede a la arteriografía, embolizando selectivamente, mediante colis, la arteria renal. Tres días después se realiza nefrectomía radical por vía anterior, apreciándose gran masa tumoral dependiente del riñón derecho, que no afecta al músculo psoas.

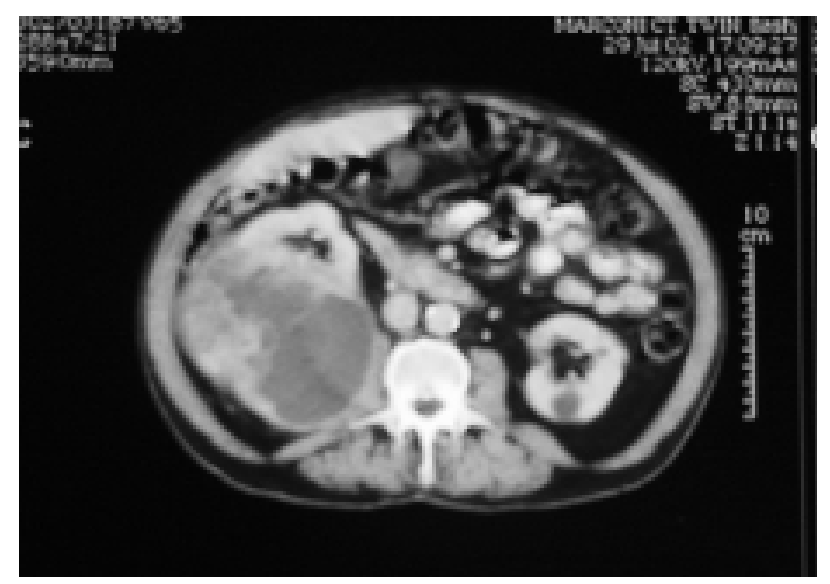

FIGURA 1. Tumoración hemorrágica retroperitoneal dependiente de riñón derecho.

Histológicamente, a nivel macroscópico se objetiva masa tumoral de 10 centímetros de diámetro que afecta grasa perirrenal sin afectación de los sistemas excretor ni venoso. Microscópicamente es un tumor maligno fusocelular renal muy indiferenciado, tipo sarcomatoide grado 4 de Fuhrman, con infiltración perirrenal pero sin existir afectación de los sistemas excretor y venoso; pT3 G3 NO MO (Figs. 2 y 3). Tras la cirugía el paciente evoluciona favorablemente siendo dado de alta al décimo día post-operatorio.

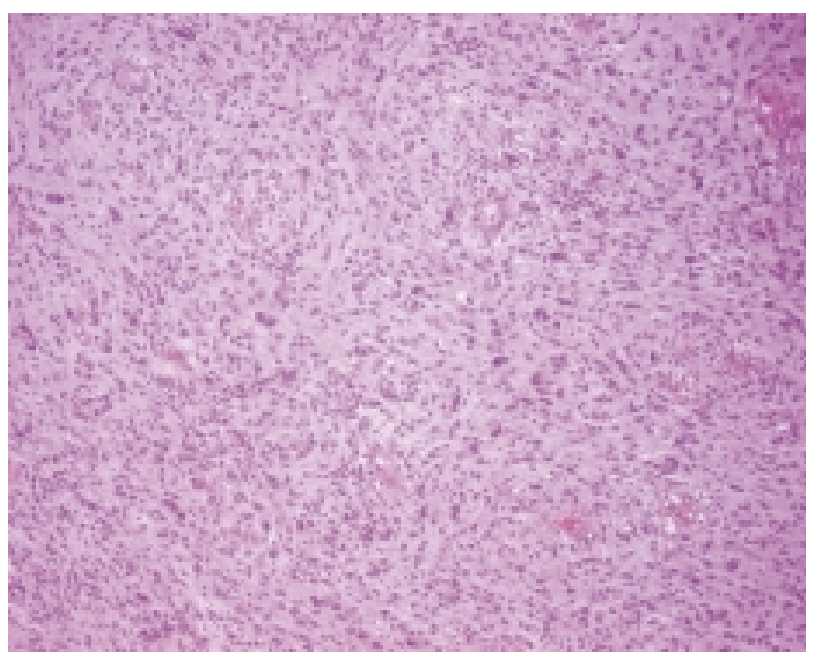

FIGURA 2. Microscopia óptica con bajo aumento de la lesión tumoral.

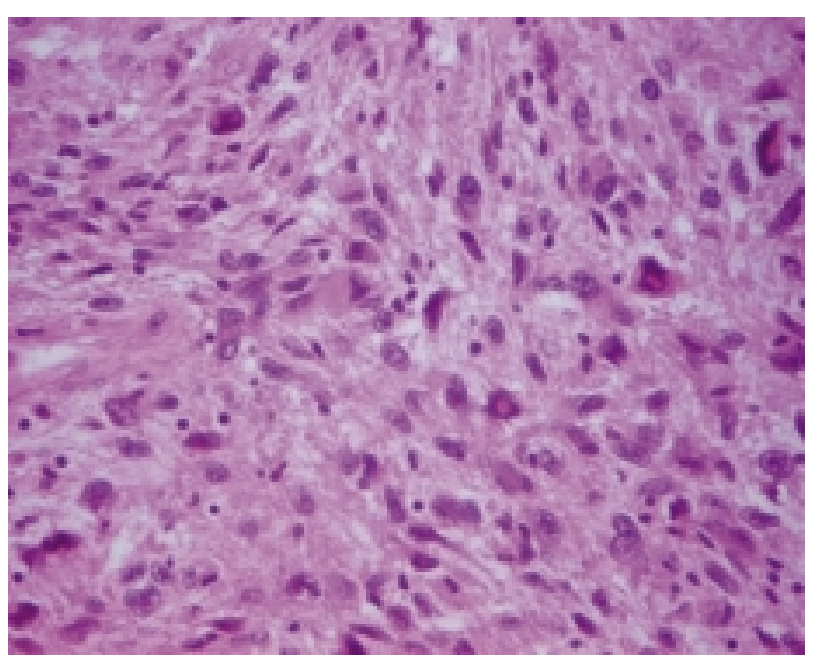

FIGURA 3. Celularidad fusocelular en microscopia óptica de la lesión tumoral.

En la primera revisión, a los seis meses de la intervención, en la TAC de control (Figs. 4 y 5), se evidencian dos masas quísticas heterogéneas en espacio retroperitoneal; la primera adyacente al músculo psoas de unos 12 centímetros de diámetro, con probable infiltración hepática y de colon ascendente; la segunda de 10 centímetros adyacente al músculo psoas. Se realiza punción aspiración por aguja fina (P.A.A.F.) ecodirigida siendo el resultado de metástasis de carcinoma renal. La gammagrafía ósea de cuerpo completo no evidenció lesiones metastásicas.

A las 72 horas de la P.A.A.F. el paciente presenta mareo, distensión abdominal y anemia, 


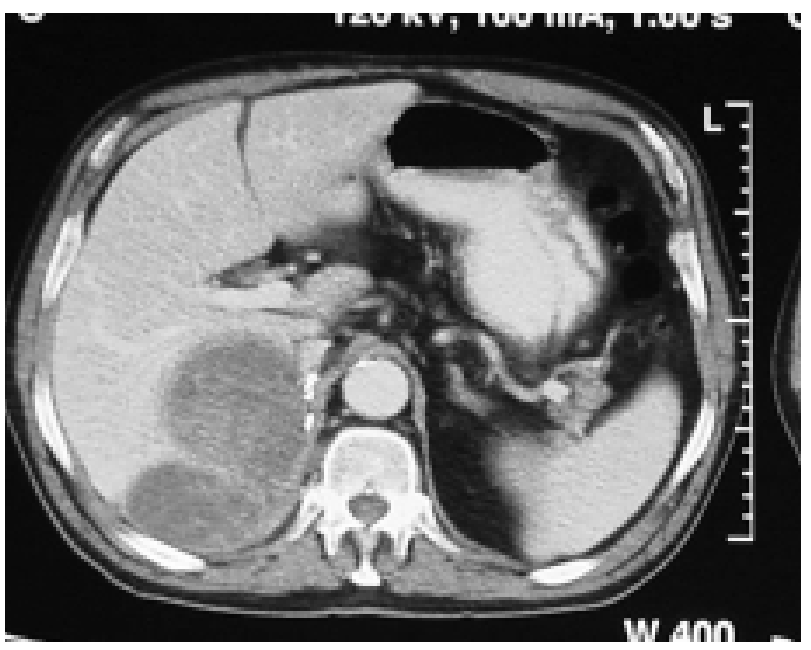

FIGURA 4. Masa heterogénea retroperitoneal.

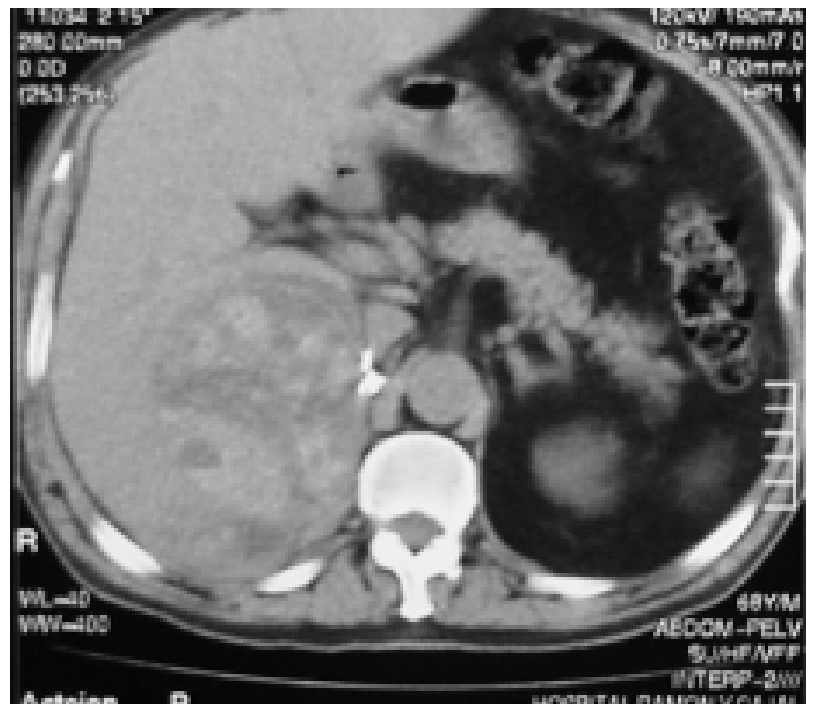

FIGURA 5. Masa heterogénea retroperitoneal con áreas de sangrado.

realizándose analítica apreciándose la pérdida de $5 \mathrm{gr} / \mathrm{dl}$ de hemoglobina respecto al alta. La TAC en ese momento evidenció gran masa retroperitoneal, con intenso componente hemorrágico (Fig. 6). Se realiza laparotomía exploradora, evacuando hematoma retroperitoneal, y realizando exéresis de tumoración quística hemorrágica, adherida al lóbulo caudado del hígado, que en la biopsia intraoperatoria se informa como tumoración maligna fusocelular de alto grado, parcialmente quística, compatible con recidiva de tumor renal, la evolución post-operatoria el paciente presenta nuevo hematoma retroperitoneal.

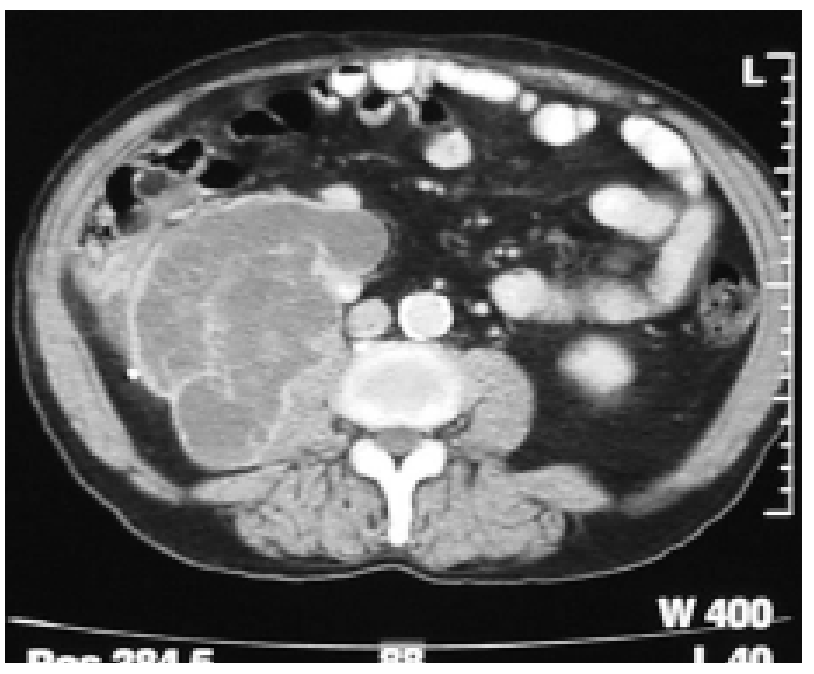

FIGURA 6. Lesión hemorrágica retroperitoneal.

\section{DISCUSIÓN}

El carcinoma de células renales representa el $3 \%$ de los tumores del hombre; siendo el carcinoma de células renales el $75 \%$ aproximadamente de los tumores malignos de riñón, mientras que los tumores derivados del tejido mesenquimal no representan más del $3 \%^{1}$. El carcinoma renal sarcomatoide fue descrito por Farrow por primera vez en 1968 representando entre el 1 y el 6,5\% de los carcinomas de células renales, según las series $^{4-7,11,16}$; en España el primer caso fue publicado en 1978 por Figueiredo ${ }^{13}$ et al. En la bibliografía nacional se han descrito 53 casos $^{4,5,7,11-}$ $14,16,17,22,23$. La incidencia es mínimamente superior en el hombre que en la mujer siendo la sexta década de la vida la de mayor prevalencia. La edad de aparición más temprana reportada en la bibliografía internacional son 29 años $^{18}$.

El carcinoma de células renales deriva de las células del tubo contorneado proximal. Según las características microscópicas de la célula renal, se distinguen tres variantes citológicas: la célula clara (la más frecuente), siendo la variante celular granular y la fusiforme menos usuales. Cuando la estirpe citológica predominante es la célula fusiforme, nos encontramos ante la variante sarcomatoide del carcinoma renal, llamada así, debido a la similitud entre la disposición ahusada de las células de la variante sarcomatoide y las distintas formas celulares que pueden presentar los sarcomas ${ }^{2}$. 
Histológicamente estos tumores se caracterizan por presentar doble población celular, las células claras típicas del carcinoma renal y las células fusocelulares, acompañándose estas últimas, habitualmente de diversas áreas de necrosis. El diagnóstico histopatológico de carcinoma fusocelular, exige objetivar componente epitelial en el tumor, o demostrar el origen epitelial de las células fusocelulares ${ }^{15}$. El componente fusocelular de estos tumores puede asemejar a fibrosarcomas y fibrohistiocitomas, lo cual lleva hacer diagnóstico diferencial entre carcinoma sarcomatoide y sarcoma renal. El origen histológico del carcinoma fusocelular es controvertido, Farrow $^{1}$, sugiere que el origen es la metaplasia de las células del adenocarcinoma mientras que Benngton ${ }^{2}$, mantiene que es una metaplasia del estroma renal, Cecil defiende que un mismo tumor prolifera hacia estirpe epitelial y mesenquimal ${ }^{3}$. La diferencia existente entre las células sarcomatoides y las estromales, es la formación en las primeras de membrana basal en forma de ribete de cepillo y la presencia de desmosomas. El estudio inmunohistoquímico facilita el diagnóstico, ya que permite identificar citoqueratinas, que nos orientan hacia el origen epitelial del componente fusocelular; siendo la mayoría positivos para queratina, vimentina, y URO I, II, II, IV, V. La gradación histológica según el método de Fuhrman, de estos tumores suele ser grado III o IV, en más del 70\% de los casos ${ }^{4-6,7,11}$. En ocasiones el componente sarcomatoide del tumor se diferencia hacia cartílago, músculo esquelético o hueso ${ }^{3,8,9}$.

Como en otros casos de la literatura el síndrome constitucional intenso y el dolor lumbar con signos de sangrado son el dato clínico más destacable $^{4,5}$. Otro sintoma usual es la hematuria. Al igual que en nuestro paciente, el signo más habitual encontrado es la masa abdominal palpable ya que la enfermedad suele estar avanzada locorregionalmente en el momento del diagnóstico ${ }^{5-7,11}$. La recidiva precoz, el sangrado retroperitoneal, la infiltración hepática, y el fallecimiento pocos meses después de la segunda intervención, como en nuestro caso, ha sido descrita en un paciente de la serie de Lecumberri et al. ${ }^{15}$, en el cual un paciente falleció a los tres meses de la recidiva. Las principales localizaciones de metastásicas son el hueso y pulmón ${ }^{19,20}$; siendo el lugar más frecuente de recidiva a nivel locorregional.
El diagnóstico definitivo es el patológico. Analíticamente, la elevación de la velocidad de sedimentación y anemia, son los datos más frecuentes, seguido por alteraciones de la función hepática ${ }^{5,7,11,18}$. En la T.A.C., son indistinguibles del adenocarcinoma renal, asemejando una masa de características heterogéneas que nace del parénquima renal, con áreas de necrosis, hemorragia y frecuentemente infiltración de estructuras adyacentes. La angiografía presenta un patrón hipervascular ${ }^{10}$. Se ha descrito un caso en el que aparecía una masa calcificada y avascular que necesitó el diagnóstico diferencial con un sarcoma renal ${ }^{4}$. El diagnóstico diferencial debe realizarse con sarcomas, uroteliomas y en pacientes jóvenes con tumor de Wilms del adulto.

El pronóstico es malo, con una elevada frecuencia de recidiva tumoral a nivel regional, con una supervivencia media de 6 meses, e inferior al año en la mayoría de los casos, con una supervivencia a los cinco años no mayor del $2 \%{ }^{5,7,11,16,17}$. El pronóstico está íntimamente relacionado con el grado celular y nuclear (pleomorfismo, aneuploidía), estadio, porcentaje de celularidad fusocelular del tumor, y extensión de la necrosis ${ }^{6}$. Estudios por citometría relacionan el perímetro nuclear y el volumen nuclear con la supervivencia $^{11}$. El estadio es el factor más importante en la supervivencia $^{6}$. El factor más importante en el pronóstico y evolución de la enfermedad es el estadio (los casos de mayor supervivencia o libres de enfermedad de las distintas series, son pacientes con estadios I o II), seguido de la extensión de la necrosis y la proporción del componente sarcomatoide ${ }^{6}$.

Al tratamiento de elección es la nefrectomía radical. Terapias coadyuvantes como la inmunoterapia (interleukina 2 , interferon alfa), quimioterapia (vinblastina, doxirrubicina) o radioterapia, parecen ofrecer resultados pobres, pero abren una línea para la terapia de este tumor ${ }^{5,20,21}$. Culine et cols. ${ }^{20}$, en una serie de 14 pacientes trató 4 pacientes con inmunoterapia (interferon) no existiendo respuesta en ellos; 9 con quimioterapia (doxirrubicina) entrando tres de ellos en remisión (siendo la supervivencia media de 38 meses); y uno no recibió tratamiento coadyudante, en el cual fue necesario la exéresis de una metástasis pulmonar y que en la actualidad sigue 
vivo. No demostrándose remisión completa ni con la inmunoterapia y sólo en tres pacientes en tratamiento quimioterapéutico, aún así los pacientes tratados con inmunoterapia la supervivencia media fue de 20 meses más que los que se les administró quimioterapia. Cangiano ${ }^{21}$ en su serie, informó que el riesgo relativo de fallecimiento con dosis altas de interleukina 2 es 10,4 veces menor que si el tratamiento se administra a bajas dosis; también trató un paciente con interferon alfa siendo éste el de mayor supervivencia.

\section{CONCLUSIONES}

El carcinoma sarcomatoide de riñón, es un tumor con una incidencia del 1 al 6,5\% de los tumores renales. La clínica es similar en la mayoría de los casos, siendo el dolor en flaco, el síndrome constitucional y la sensación de masa los más habituales. El diagnóstico definitivo es el patológico pero la TAC y la ecografía son las pruebas diagnósticas de elección. La media de la supervivencia es de seis meses, siendo el factor pronóstico más importante el estadio tumoral. El tratamiento de elección es la nefrectomía radical, no existiendo en la actualidad resultados concluyentes sobre el valor de la terapia coadyuvante (inmunoquimioterapia) en este tipo de tumor.

\section{REFERENCIAS}

1. FARROW GM, HARRISON EG JR, UTZ DC. Sarcomas and sarcomatoid and mixed malignant tumors of the kidney in adults. I- II- III. Cancer 1968 sep; 22 (3): 545-563.

2. BENNGTON JL.: Tumors of the kidney, renal pelvis and ureter. Atlas of tumors pathology. $2^{\mathrm{a}}$ ed: serie 12pp 229. AFIP. Washinton.

3. CECIL B, WOODARD B, MICKEY DD, PIZZO S.: Renal carcinosarcoma. Ultrastructure and transplantation into athymic mice. Arch Pathol Lab Med 1980 may; 104 (5): 276-279.

4. HERRERA PUERTO J, SOLER FERNÁNDEZ J, DOMÍNGUEZ BRAVO C.: Carcinoma renal sarcomatoide: presentación de un caso. Actas Urol Esp 1989 nov-dec; 13 (6): 467-469.

5. GÓMEZ VEGAS A, BLÁZQUUZZ IZQUUIERDO J, BLANCO GONZÁLEZ J.: Carcinoma renal sarcomatoide. Arch Esp Urol 1998 mar; 51 (2): 154-158.

6. RO JY, AYALA AG, SELLA A.: Sarcomatoid renal cell carcinoma: clinicopathologic. A study of 42 cases. Cancer 1987 feb 1; 59 (3): 516-526.

7. BORDALBA JR, GIL VERNET A, CONDOM E.: Carcinoma renal sarcomatoide. Actas Urol Esp 1991 sep-oct; 15 (5): 478-480.
8. ITOH T, CHIKAI K, OTA S, NAKAGAWA T.: Chromophobe renal cell carcinoma with osteosarcoma-like differentiation. Am J Surg Pathol 2002 oct; 26 (10): 1.358-1.362.

9. MACKE RA, HUSSAIN MB, IMRAY TJ.: Osteogenic and sarcomatoid differentiation of a renal cell carcinoma. Cancer 1985 nov 15; 56 (10): 2.452-2.457.

10. SHIRKHODA A, LEWIS E.: Renal sarcoma and sarcomatoid renal cell carcinoma: CT and angiographic features. Radiology 1987 feb; 162 (2): 353-357.

11. MARTÍNEZ JABALOYAS JM, RUIZ CERDÁ JL, SANZ CHINESTA S.: Carcinoma renal sarcomatoide. Actas Urol Esp 1996 oct; 20 (9): 800-805.

12. LOZANO ORTEGA JL, MAYAYO E, REY A.: Carcinoma renal sarcomatoide. A propósito de un caso. Arch Esp Urol 1986 apr; 39 (3): 200-203.

13. FIGUEIREDO GODOY L, NOGUEIRA MARCH JL.: Carcinoma renal sarcomatoide. Arch Esp Urol 1978 jul-aug; 31 (4): 397-406.

14. LEUCUMBERRI CASTANOS D, PODILLA NIEVA J, ALBISU TRISTÁN A.: Carcinoma renal sarcomatoide. Arch Esp Urol 1998 oct; 51 (8): 772-775.

15. ALGABA F.: Atlas de patología de los tumores urogenitales. Editorial Pulso, $1^{\underline{a}}$ edición. Barcelona 1991: 54.

16. GUTIÉRREZ BAÑOS JL, MARTÍN GARCÍA B, HERNÁNDEZ RODRÍGUEZ R.: Carcinoma renal sarcomatoide. Arch Esp Urol 1993 apr; 46 (3): 199202.

17. ARRURTI IZCO JM, BERNE MANERO JM, TRALLERO CATEVILLA MJ.: Carcinoma renal sarcomatoide. Presentación de dos casos. Arch Esp Urol 2003; 56 (1): 71-74.

18. GARCÍA MEDIERO JM, ALONSO DORREGO JM, NÚÑEZ MORA C.: Carcinoma renal sarcomatoide de temprana aparición. Aportación de un nuevo caso y comparación del mismo con otro de aparición tardía. Arch Esp Urol 2002; 55 (7): 843-847.

19. DE PERALTA-VENTURINA M, MOCH H, AMIN M.: Sarcomatoid renal cell carcinoma: a study of 101 cases. Am J Surg Pathol 2001; 25 (3): 275-284.

20. CULINE S, BEKRADDA M, TERRIER-LACOMBE MJ.: Treatment of sarcomatoid renal cell carcinoma: is there a role for chemotherapy?. Eur Urol 1995; 27 (2): 138-141.

21. CANGIANO T, LIAO J, NAITOH J, DOREY F.: Sarcomatoid renal cell carcinoma: biologic behavior, prognosis, and response to combined surgical resection and immunotherapy. J Clin Oncol 1999 feb; 17 (2): 523-528.

22. MUÑOZ VÉLEZ D, BENEJAM GUAL JM, GARCÍAMIRALLES GRAVALOS R.: Carcinoma renal sarcomatoide localizado. ¿Qué factores pueden prever su evolución?. Actas Urol Esp 2002; 26 (1): 60-65.

23. LLARENA IBARGUREN R, ZABALA EGURROLA JA, MARÍN LAFUENTE JC.: Carcinoma renal sarcomatoide. Arch Esp Urol 1990 may; 43 (4): 409-411.

Dr. I. Gómez García

C/ Costa Brava, 9 - portal 3, 1ㅇ D

28034 Madrid

(Trabajo recibido el 19 mayo de 2003) 